ARISTOTLE'S POETICS: THE ARGUMENT 



\title{
ARISTOTLE'S POETICS: THE ARGUMENT
}

\author{
BY \\ GERALD F. ELSE
}

Published in coöperation with the

State University of Iowa

HARVARD UNIVERSITY PRESS

Cambridge, Massachusetts

1957 
All rights reserved

Distributed in Great Britain by
Oxford University Press, London

Library of Congress Catalog Card Number A 57-8620

PRINTED BY DE MEESTER BROS.

WETTEREN - BELAIUM 
UNIVERSITATIBUS HARVARDIANAE • IOWENSI •

ILLI ALMAE MATRI - CARIS AMBABUS •

QUARUM ALTERA ME IUVIT LONGUM OPUS INGREDIENTEM ALTERA PERFICIENTEM

$\Sigma Y N \triangle I K O N$ 
